\title{
Roadblocks in the Delivery of Cancer Care in India during COVID Pandemic
}

\author{
Prerana Nagabhushana ${ }^{1} \oplus$, Vanita Suri ${ }^{2}$, Subhash C Saha ${ }^{3}$
}

\begin{abstract}
The coronavirus disease (COVID) pandemic has impacted the health sector in massive proportions. Perhaps the worst affected aspect is that of oncological care. Cancer patients continue to suffer silently due to nonavailability of consultations and shortage of operating rooms. We need to brace ourselves for the impact of this backlog of nearly 18 months of neglected care of such patients.

Keywords: Ca cervix, Ca ovary, Cancer, Cancer cervix, Chemotherapy, Malignancy, Mortality, Ovarian carcinoma, Rural, Screening.

Journal of South Asian Federation of Obstetrics and Gynaecology (2021): 10.5005/jp-journals-10006-1966
\end{abstract}

The global impact of the unleashing of the coronavirus is tremendous and far-reaching. ${ }^{1}$ Across continents, countries, races, and religions, people continue to suffer today still, thanks to the myriad mutant avatars of this faceless enemy. As a combative measure to curb the spread of the pandemic, many governments enforced nationwide lockdown with severe restriction of interand intrastate travel and trade. Prioritization of coronavirus disease-2019 (COVID-19) treatment in terms of health personnel and resources has pushed other important aspects of healthcare to the backseat. As the pandemic blatantly refuses to abate, sucking us dry of patience and resources, many non-COVID patients continue to suffer silently.

Mrs. M, a 39-year-old mother of two, resident of a remote village in the hilly state of Himachal Pradesh, noticed a lump in her right breast and vague abdominal pain a year ago. Due to the loss of monthly income and transport restrictions imposed by the lockdown, she could not reach healthcare facilities in time. Instead, she went to a local quack and took desi medications for almost 6 months. Today, she has an advanced-stage breast cancer which is inoperable in addition to a massive ovarian malignancy! By the time she reached a tertiary-level cancer center after multiple referrals, her disease had progressed and her morale broken both by the diagnosis and its implications on her family.

Of the many aspects of health care that took a hit during the last year, cancer care, by far, seems the worst affected. ${ }^{2,3}$ For the past 18 months, cancer screening programs have faced many setbacks. Many cancer patients, like Mrs. M, are unable to reach the appropriate health facility in time either due to financial constraints or other logistic factors, which delays their timely diagnosis. In addition to being burdened by the diagnosis of cancer, these patients must now additionally suffer due to delay in surgery of operable disease and fear of acquiring COVID infection, especially postchemotherapy. Being an extensive and expensive treatment with questionable prognosis, cancer care exhausts their families financially, physically and emotionally. The all-important aspect of supportive care such as emotional support, counseling, and group therapy is being largely denied to these patients today. Many of them are lost to follow-up and continue to suffer quietly (Fig. 1).

With the abatement of the second wave and the government approval for universal COVID vaccination, hope seems to be budding among these patients. As active COVID cases continue to fall, many
${ }^{1-3}$ Department of Obstetrics and Gynecology, Postgraduate Institute of Medical Education and Research, Chandigarh, India

Corresponding Author: Prerana Nagabhushana, Department of Obstetrics and Gynecology, Postgraduate Institute of Medical Education and Research, Chandigarh, India, Phone: +91 8277116563, e-mail:nprerana1993@gmail.com

How to cite this article: Nagabhushana P, Suri V, Saha SC. Roadblocks in the Delivery of Cancer Care in India during COVID Pandemic. J South Asian Feder Obst Gynae 2021;13(5):364-365.

Source of support: Nil

Conflict of interest: None

centers have now restarted oncosurgical procedures. It is imperative to prioritize treating these long-suffering patients despite the pandemic and offer them supportive care as well. Physicians working at the first-reach hospitals must refer these patients timely to higher centers. Hospitals must urgently establish fast-track protocols for diagnosing and treating such patients as early as possible. In their

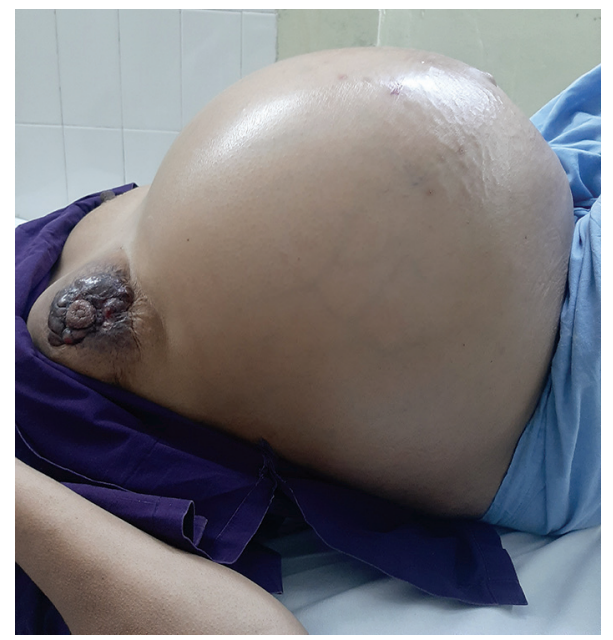

Fig. 1: A mother of two, sole bread-winner of her family, came to us with advanced ovarian and breast cancer due to delay in care for almost 2 years! She had a massive abdominal mass and inoperable breast cancer 
interests, hospital policy must dictate resources not be diverted from cancer care and follow-up. In addition, we must also offer them counseling and involve supportive staff in their follow-up. Cancer patients and their caregivers must remain focused in face of the upcoming third wave and brace for clearing the backlog of cases. In conclusion, policy makers must emphasize on continued treatment indeed with doubled resources of noncommunicable diseases such as cancer.

\section{OrCID}

Prerana Nagabhushana $\odot$ https://orcid.org/0000-0002-4728-8721

\section{References}

1. Available from: https://www.who.int/news/item/13-10-2020-impactof-covid-19-on-people\%27s-livelihoods-their-health-and-our-foodsystems

2. Jazieh AR, Akbulut H, Curigliano G, et al. International Research Network on COVID-19 Impact on Cancer Care. Impact of the COVID-19 pandemic on cancer care: a global collaborative study. JCO Glob Oncol 2020;6:1428-1438. DOI: 10.1200/GO.20.00351.

3. Ranganathan P, Sengar M, Chinnaswamy G, et al. National Cancer Grid of India. Impact of COVID-19 on cancer care in India: a cohort study. Lancet Oncol 2021:S1470-2045(21)00240-0. DOI: 10.1016/ S1470-2045(21)00240-0. 